\title{
Molecular Cloning of Insulin-like Growth Factor-I (IGF-I) and IGF-II Genes of Marine Medaka (Oryzias dancena) and Their Expression in Response to Abrupt Transfer from Freshwater to Seawater
}

\author{
Yue Jai Kang and Ki Hong Kim* \\ Department of Aquatic Life Medicine, Pukyong National University, \\ Busan 608-737, Korea
}

\begin{abstract}
Growth hormone $(\mathrm{GH})$ is known as one of the main osmoregulators in euryhaline teleosts during seawater (SW) adaptation. Many of the physiological actions of GH are mediated through insulin-like growth factor-I (IGF-I), and the GH/IGF-I axis is associated with osmoregulation of fish during SW acclimation. However, little information is available on the response of fish IGF-II to hyperosmotic stress. Here we present the first cloned IGF-I and IGF-II cDNAs of marine medaka, Oryzias dancena, and an analysis of the molecular characteristics of the genes. The marine medaka IGF-I cDNA is $1,340 \mathrm{bp}$ long with a $257 \mathrm{bp} 5^{\prime}$ ' untranslated region (UTR), a 528 bp 3' UTR, and a 555-bp open reading frame (ORF) encoding a propeptide of 184 amino acid (aa) residues. The full-length marine medaka IGF-II cDNA consists of a 639 bp ORF encoding 212 aa, a 109 bp 5' UTR, and a 416 bp 3' UTR. Homology comparison of the deduced aa sequences with other IGF-Is and IGF-IIs showed that these genes in marine medaka shared high structural homology with orthologs from other teleost as well as mammalian species, suggesting high conservation of IGFs throughout vertebrates. The IGF-I mRNA level increased following transfer of marine medaka from freshwater (FW) to SW, and the expression level was higher than that of the control group, which was maintained in FW. This significantly elevated IGF-I level was maintained throughout the experiment (14 days), suggesting that in marine medaka, IGF-I is deeply involved in the adaptation to abrupt salinity change. In contrast to IGF-I, the increased level of marine medaka IGF-II mRNA was only maintained for a short period, and quickly returned a level similar to that of the control group, suggesting that marine medaka IGF-II might be a gene that responds to acute stress or one that produces a supplemental protein to assist with the osmoregulatory function of IGF-I during an early phase of salinity change.
\end{abstract}

Key words: cDNA cloning, Hyperosmotic stress, Insulin-like growth factor-I (IGF-I), IGF-II, Marine medaka (Oryzias dancena)

\section{Introduction}

For aquatic organisms, salinity is a crucial factor for maintaining normal physiological processes. Euryhaline teleosts, which can tolerate wide variations in salinity without compromising life processes, are equipped with effective osmoregulatory mechanisms against dramatic environmental salinity changes. Some species of the genus Oryzias including Japanese medaka, O. latipes, a well-known experimental model fish with a genomic database

\footnotetext{
*Corresponding author: khkim@pknu.ac.kr
}

(Wittbroadt et al., 2002), are good model species for analyzing osmoregulatory mechanisms in teleosts. Among them, the marine medaka, O. dancena, a fish species inhabiting estuarine waters (Roberts, 1998), has been reported to have better tolerance against hyperosmotic stress than O. latipes (Inoue and Takei, 2002, 2003; Kang et al., 2008).

Growth hormone $(\mathrm{GH})$ is known as one of the main osmoregulators in euryhaline teleosts during seawater (SW) adaptation (McCormick, 1995). Many of the physiological actions of $\mathrm{GH}$ are mediated through insulin-like growth factor-I (IGF-I) (Froesch et al., 1985; Butler and Le Roith, 2001), and the GH/IGF-I 
axis is associated with osmoregulation of fish during SW acclimation (Madsen and Bern, 1993; Sakamoto and Hirano, 1993; McCormick, 1995, 1996; Kalujnaia et al., 2007; Meier et al., 2009). As in mammals, teleosts possess IGF-I and IGF-II (Reinecke et al., 2005). The telesotean IGF-I is involved in osmoregulation as well as many developmental, physiological, and immunological responses (Reinecke, 2006; Yada, 2007). However, although the expression of the IGF-II gene was demonstrated to be GH-dependent in several fish species, such as rainbow trout and redbanded seabream (Shamblott et al., 1995; Ponce et al., 2008), little information is available on the response of fish IGF-II to hyperosmotic stress.

Here we report the first cloned IGF-I and IGF-II cDNAs of marine medaka and present an analysis of the molecular characteristics of the genes. In addition, to determine whether the IGFs respond to acute hyperosmotic stress, time-course changes of IGF-I and IGF-II transcripts levels were examined after abrupt transfer of marine medaka from freshwater (FW) to SW.

\section{Materials and Methods}

\section{Fish}

Marine medakas (O. dancena) were obtained from brood stock maintained at the Institute of Marine Living Modified Organisms (IMLMO), Pukyong National University. Yolk-sac absorbed fry were held in two $50 \mathrm{~L} \mathrm{FW}$ aquaria and acclimated for 2 weeks. Throughout the study, the water temperature was maintained at $27 \pm 1^{\circ} \mathrm{C}$. After acclimation, fish were transferred directly from $\mathrm{FW}$ to $\mathrm{SW}$ or from $\mathrm{FW}$ to FW (control group), and sampled at $0,3,6,12,24,72$, 168 ( 7 days) and 336 (14 days) h after transfer. Five fish were randomly collected at each time point to analyze the mRNA levels of IGF-I and IGF-II.

\section{Gene cloning}

Total RNA was extracted from the whole body of fish using the RNAiso reagent (Takara Bio, Tokyo, Japan) according to the manufacturer's instruction. A $1-\mu \mathrm{g}$ aliquot of purified total RNA was incubated with $1 \mu \mathrm{L}$ of random primer $(0.5 \mu \mathrm{g} / \mathrm{mL})$ (Promega, Madison, WI, USA) at $80^{\circ} \mathrm{C}$ for $10 \mathrm{~min}$, and further incubated at $42^{\circ} \mathrm{C}$ for $60 \mathrm{~min}$ in a reaction mixture containing $4 \mu \mathrm{L}$ of $5 \times$ reaction buffer, $4 \mu \mathrm{L}$ of $10 \mathrm{mM}$ dNTP mix (Takara Bio), $1 \mu \mathrm{L}$ of M-MLV reverse transcriptase (Promega), and $0.5 \mu \mathrm{L}$ of RNase inhibitor (Promega) in a final reaction volume of $20 \mu \mathrm{L}$.
Two degenerate oligonucleotides, IGF-I F: 5'GTSTGYGGRGASMGRGGCTTTTATTTCA-3' and IGF-I R: 5'-GGGGGMAGRAAYTAYCGVATKTA G-3', were used to obtain the marine medaka IGF-I cDNA fragment by PCR. Using another two degenerate oligonucleotides (IGF-II F: 5'-GGAGARCT GGTRGAYGCGCTRCAGTTYGTSTG-3' and IGFII R: 5' - CRGMGRCTMCGRAGRGGYRTCCC-3'), a partial fragment of marine medaka IGF-II cDNA was obtained by PCR. The amplified PCR product was run on an agarose gel $(1.5 \%)$ and visualized by ethidium bromide (Et-Br) staining. The fragment was purified using a gel purification kit (Cosmo Genentech, Seoul, Korea) and subcloned into a pGEM-T easy vector (Promega). Several clones were sequenced using two vector primers (T7 and SP6). The sequence was submitted to GenBank (http:// www.ncbi.nlm.nih.gov/blast) to examine the homology with previously known IGF sequences.

To obtain full ORF sequences of IGF-I and IGFII, 5'-RACE (primer 1: 5'-CAACGGGCTACGGTC CCAATGC-3' in IGF-I; primer 2: 5'-CTGTGCCAA ACCCACCAAGTCCGAAAG-3' in IGF-II; primer 3: 5'- AACCAAAACCCGCGTCTGCCATG-3' in IGF-II) and 3'-RACE (primer 4: 5'-GAGGCAACAA CGGGGGCAGAAA-3' in IGF-I; primer 5: 5'CCCTTTGCACAGACAAGAGTTTGAGGG-3' in IGF-II) PCR reactions were performed separately using the SMART RACE cDNA Amplification Kit (Clontech, Mountain View, CA, USA) according to the manufacturer's instructions. All RACE PCR products were purified using a spin column (Labopass; Cosmo Genentech), cloned into a pGEM-T easy vector (Promega), and sequenced. Multiple sequence alignments were generated using the CLUSTALW 1.8 program.

\section{Semiquantitative RT-PCR}

The expression of IGF-I and IGF-II in response to hypersalinity stress was analyzed using semiquantitative RT-PCR. Fish were anesthetized with tricaine methanesulfonate (MS222; Sigma, St. Louis, MO, USA), and total RNA was extracted from the whole body using RNAiso reagent, then reversetranscribed to cDNA as described above to be used for the gene expression assay. Primers used for amplification of each gene were as follows: IGF-IF (5'-CAACGGGCTACGGTCCCAATGC-3') and IGF-I-R (5'-GAGGCAACAACGGGGGCAGAAA3') for IGF-I, IGF-II-F (5'-ATGGAGAGCCCGCA AAGACTCGG-3') and IGF-II-R (5'-CTCAACCT GCTAGAGCAGTACTGTGCCAA-3') for IGF-II, Actin-F (5'-ATGGAGAAGATCTGGCATCACACC 
TTCTAC-3') and Actin-R (5'-GGCTGGCAGAGA TTTGACAGATTACCTGAT-3') for the $\beta$-actin gene (internal control). PCR was performed using Takara rTaq premix and $1 \mu \mathrm{L}$ of tenfold diluted cDNA in a $20 \mu \mathrm{L}$ reaction. For normalization, the $\beta$-actin gene was used as an internal standard. The optimal PCR cycles of the selected genes were determined after PCR products taken every 2 or 3 cycles from 15 to 33 cycles, and were confirmed by agarose gel electrophoresis. PCR samples to be compared were visualized on $1 \%$ agarose gels with Et-Br staining. Images of the Et-Br stained bands were imported into the Quantity-One image analysis software implemented in Gel Doc XR imaging device (Bio-Rad, Hercules, CA, USA). The band intensity of IGF-I and IGF-II genes was normalized against that of $\beta$-actin to estimate their relative mRNA levels.

\section{Statistics}

Semiquantitative RT-PCR data were analyzed using the Student $t$-test, and significant differences were determined at $P<0.05$.

\section{Results}

\section{Complementary DNAs and deduced amino acid} sequences of marine medaka IGF-I and IGF-II

The marine medaka IGF-I cDNA was 1,340 bp long with a 257 bp 5' UTR, a 528 bp 3' UTR, and a 555 bp ORF encoding a propeptide of 184 amino acid (aa) residues (GenBank Accession No. HQ176000). Conserved domain analysis revealed that the marine medaka IGF-I had two cleavage regions, an Nterminal signal peptide (1 to $44 \mathrm{aa}$ ), and a $\mathrm{C}$ terminal E domain (113 to 184 aa), which made the propeptide into a mature peptide of 68 aa residues. The mature peptide consisted of domains B (45 to 73 aa), C (74 to $83 \mathrm{aa}$ ), A (84 to $104 \mathrm{aa}$ ), and D (105 to $112 \mathrm{aa})$. At the aa level, marine medaka IGF-I showed high similarity with the IGF-Is of other fish species (64$87 \%$ ). Mouse (Mus musculus) and human (Homo sapiens) IGF-Is showed 58\% and 59\% identity, respectively, with marine medaka IGF-I. Multiple alignments of IGF-I proteins showed that the B and A domains of the mature form IGF-I were highly conserved from fish to mammals. The signal peptide region and the $\mathrm{C}$-terminal $\mathrm{E}$ domain were highly variable according to fish species (Fig. 1).

The full-length marine medaka IGF-II cDNA consisted of a 639 bp ORF encoding 212 aa, a 109 bp 5' UTR, and a 416-bp 3' UTR (GenBank Accession No. HQ176001). Conserved domain analysis revealed that marine medaka IGF-II consisted of a signal peptide region ( 1 to $44 \mathrm{aa}$ ) in the $\mathrm{N}$-terminal region, the domains of mature forms (B, 45 to 76 aa; $\mathrm{C}, 77$ to 87 aa; A, 88 to 108 aa; D, 109 to 114 aa) in the middle region, and the $\mathrm{C}$-terminal $\mathrm{E}$ domain (115 to 212 aa). When comparing deduced aa sequences, marine medaka IGF-II showed high similarity (6679\%) with previously reported teleostean IGF-II genes of other fish. The aa identity of marine medaka IGF-II with mouse IGF-II was $51 \%$ and that with human IGF-II was $48 \%$. Multiple alignment of IGF-II proteins showed a similar pattern to that of IGF-I (Fig. 2).

Time-course expression of IGF-I and IGF-II transcriptions in response to hyperosmotic stress

The transcription of marine medaka IGF-I in response to abrupt transfer of fish from FW to SW had significantly decreased at $6 \mathrm{~h}$ post-transfer and then was restored to the level of the control group at $12 \mathrm{~h}$ post-transfer (Fig. 3A). The expression level of IGF-I in the group that underwent hyperosmotic stress was higher than that of the control group from $24 \mathrm{~h}$ to the end of the experiment (14 days posttransfer). The transcription of marine medaka IGF-II had also significantly decreased at $6 \mathrm{~h}$ post-transfer. The expression of IGF-II had acutely increased at 12 and $24 \mathrm{~h}$ post-transfer, and then returned to a level similar to that of the control group toward the end of the experiment (Fig. 3B).

\section{Discussion}

In vertebrates, IGF-I and IGF-II are produced as preprohormones, consisting of an $\mathrm{N}$-terminal signal peptide, followed by B, C, A, D, and E domains (Jones and Clemmons, 1995). Then, mature IGFs are produced by proteolytic cleavage of signal peptide and $\mathrm{E}$ domain. In the present study, we cloned the complete cDNA of IGF-I and IGF-II of marine medaka. Conserved domain search analysis revealed that marine medaka IGF-I and IGF-II contained Nterminal and $\mathrm{C}$-terminal cleavage regions, resulting in propeptides from which functional, mature peptides consisting of $\mathrm{B}, \mathrm{C}, \mathrm{A}$, and $\mathrm{D}$ domains are produced. Homology comparison of the deduced aa sequences with other IGF-Is showed that marine medaka IGF-I shared high structural homology with orthologs from other teleosts as well as mammalian species. Marine medaka IGF-II also showed high similarity with mammalian IGF-IIs, suggesting conservation of IGFs throughout vertebrates.

Previous studies have demonstrated that teleost GH 


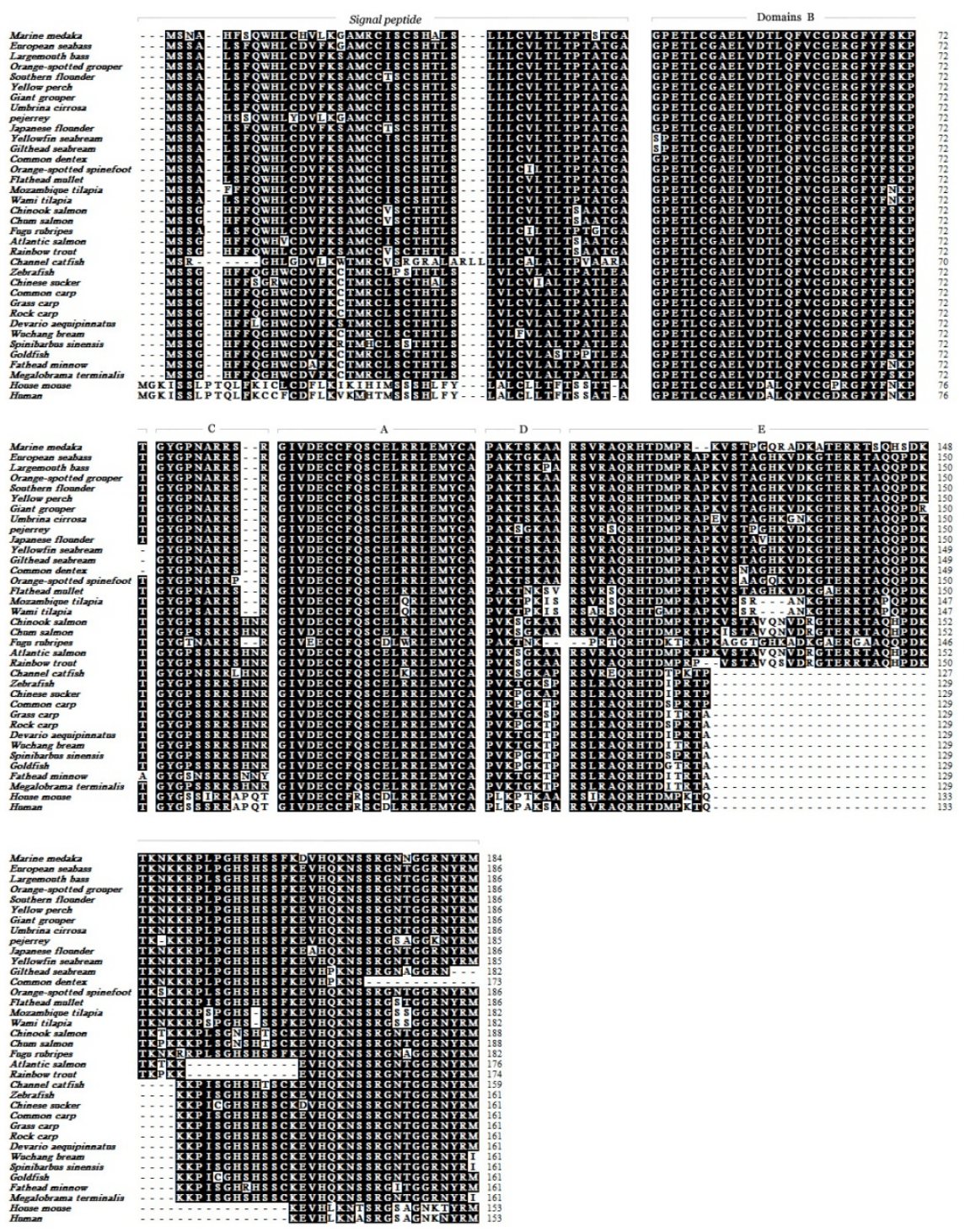

Fig. 1. Multiple alignment of deduced amino acid sequences of marine medaka IGF-I along with orthologues from other teleosteans and mammals. Amino acids identical from fish to mammalians are indicated by black boxes. Regions of signal peptides and each domain are indicated on the top line of alignment. GenBank accession number and scientific name of each species are as follows; AAV67967 Dicentrarchus labrax (European seabass), ABB70043 Paralichthys lethostigma (southern flounder), AAP93861 Perca flavescens (yellow perch), ABZ10841 Epinephelus lanceolatus (giant grouper), ABY27564 Odontesthes bonariensis (pejerrey), ABG57072 Micropterus salmoides (largemouth bass), AAY21628 Umbrina cirrosa, AAV34198 Epinephelus coioides (orange-spotted grouper), AAC62228 Paralichthys olivaceus (olive flounder), AAO47742 Siganus guttatus (orange-spotted spinefoot), AAT35826 Acanthopagrus latus (yellowfin seabream), AAR06903 Mugil cephalus (flathead mullet), ABQ52656 Sparus aurata (gilthead seabream), ABD74624 Dentex dentex (common dentex), AAA67266 Oncorhynchus tshawytscha (Chinook salmon), AAC18833 Oncorhynchus keta (chum salmon), CAA71789 Oreochromis mossambicus (Mozambique tilapia), ABN13422 Oreochromis urolepis hornorum (Wami tilapia), NP001117095 Salmo salar (Atlantic salmon), AAA49412 Oncorhynchus mykiss (rainbow trout), BAG75453 Takifugu rubripes (Fugu rubripes), NP571900 Danio rerio (zebrafish), BAA11878 Cyprinus carpio (common carp), ABU40947 Ctenopharyngodon idella (grass carp), ABB05519 Devario aequipinnatus, ACF17426 Procypris rabaudi (rock carp), AAK16727 Megalobrama amblycephala (Wuchang bream), ABH12114 Myxocyprinus asiaticus (Chinese sucker), ABE03747 Spinibarbus sinensis, AAC83444 Carassius auratus (goldfish), AAT02176 Pimephales promelas (fathead minnow), AAZ28918 Ictalurus punctatus (channel catfish), AAO89239 Megalobrama terminalis, P05017 Mus musculus (house mouse), CAA40342 Homo sapiens (Human). 

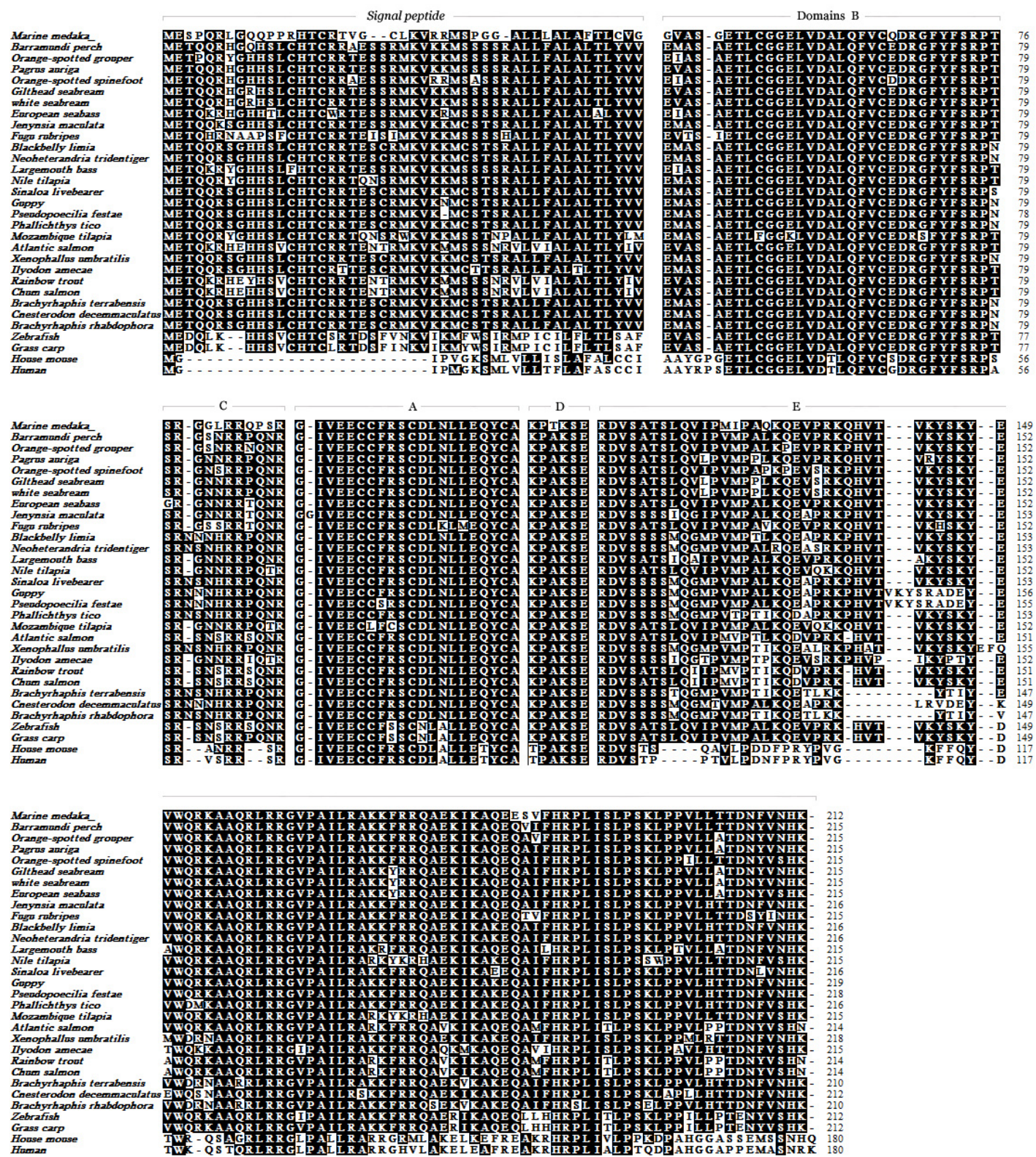

Fig. 2. Multiple alignment of deduced amino acid sequences of marine medaka IGF-II along with orthologues from other teleosteans and mammals. Amino acids identical from fish to mammalians are indicated by black boxes. Regions of signal peptides and each domain are indicated on the top line of alignment. GenBank accession number and scientific name of each species are as follows; AAB64195 Lates calcarifer (barramundi perch), AAS58520 Epinephelus coioides (orange-spotted grouper), BAF80635 Pagrus auriga, AAO47743 Siganus guttatus (orange-spotted spinefoot), AAY46224 Sparus aurata (gilthead seabream), BAF80637 Diplodus sargus (white seabream), AAW29441 Dicentrarchus labrax (European seabass), ABC60230 Jenynsia maculate, CAA17123 Takifugu rubripes (Fugu rubripes), ABC60254 Limia melanogaster (blackbelly limia), ABC60245 Neoheterandria tridentiger, ACS92730 Micropterus salmoides (largemouth bass), ABY88873 Oreochromis niloticus (Nile tilapia), ABC60234 Poeciliopsis presidionis (Sinaloa livebearer), ABC60252 Poecilia reticulata (guppy), ABC60248 Phallichthys tico, ABC60246 Pseudopoecilia festae, ABO36528 Salmo salar (Atlantic salmon), AAC17496 Oreochromis mossambicus (Mozambique tilapia), ABC60247 Xenophallus umbratilis, NP001118169 Oncorhynchus mykiss (rainbow trout), CAA65862 Oncorhynchus keta (chum salmon), ABC60229 Ilyodon amecae, ABC60249 Brachyrhaphis terrabensis, ABC60251 Cnesterodon decemmaculatus, ABC60250 Brachyrhaphis rhabdophora, NP001001815 Danio rerio (zebrafish), ABK55615 Ctenopharyngodon idella (grass carp), P09535 Mus musculus (house mouse), P01344 Homo sapiens (human). 
(A)

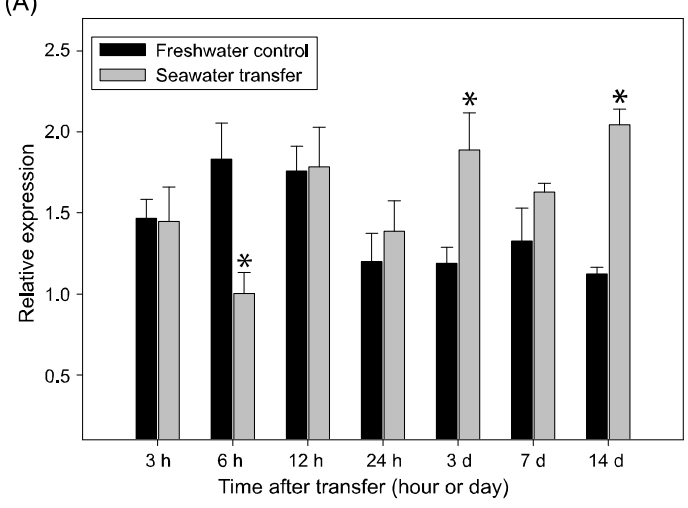

(B)

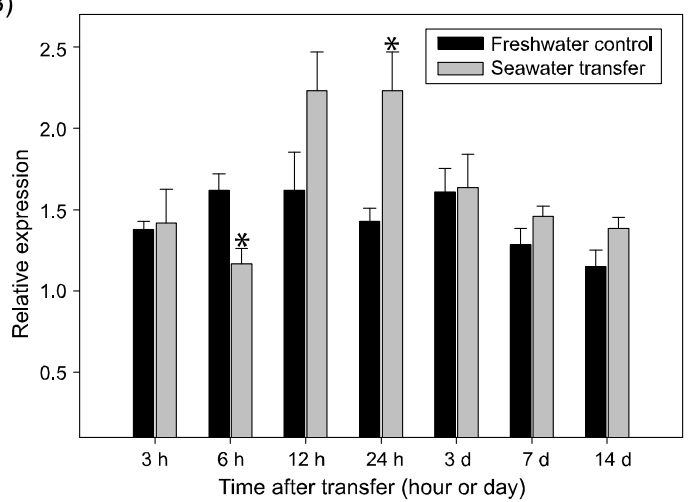

Fig. 3. Change of mRNA expression of marine medaka IGF-I (A) and IGF-II (B) genes by acute transfer from freshwater to seawater. Semi-quantitative RT-PCR was performed using cDNA synthesized using total RNA from marine medaka after transfer to seawater at various time points ranging from $3 \mathrm{~h}$ to $14 \mathrm{~d}$. Scanning densitometry analysis was done to determine the relative mRNA levels of marine medaka IGF-I or IGF-II based on the normalization against $\beta$-actin control. Histograms indicate the means of five marine medaka examinations and standard errors were indicated by $\mathrm{T}$ bars. *Significantly different from the control group $(P<0.05)$.

and IGF-I are closely associated with SW adaptation (McCormick, 2001). McCormick et al. (1991) reported that exogenous application of IGF-I resulted in improved salinity tolerance of rainbow trout after SW transfer. Sakamoto and Hirano (1993) also observed increased IGF-I mRNA levels in the gill and kidney by transfer of immature trout to $80 \% \mathrm{SW}$. Mancera and McCormick (1998) reported that killifish injected with recombinant bovine IGF-I (rbIGF-I) showed improved SW tolerance. In the present study, transferring fish from FW to SW increased the levels of IGF-I mRNA in marine medaka, and the expression level was higher than that of the control group, which was maintained in FW. The significantly higher IGF-I level was maintained throughout the experiment (14 days). This result is in accordance with previously reported responses of IGF-I levels in other fish species that were abruptly introduced to SW, suggesting that marine medaka IGF-I is deeply involved in the adaptation to abrupt salinity changes.

In contrast to IGF-I, the increased level of marine medaka IGF-II mRNA was maintained for only a short period, then quickly returned a level similar to that of the control group. This implied that acute hyperosmotic stress induces a transient increase in the expression of the IGF-II gene. This result suggests that marine medaka IGF-II might be involved in the acute stress response or that it is a supplemental protein that aids in the IGF-I osmoregulatory function during the early phases of salinity change. However, given that little information is available regarding the function of teleostean IGF-II in relation to osmoregulation, further research is needed to explain this result.

In conclusion, we cloned the cDNAs encoding marine medaka IGF-I and IGF-II and assessed the response of both genes to acute salinity change by measuring mRNA levels. This is the first report of the transient increase of IGF-II in response to acute SW transfer, and understanding the exact role of IGF-II in SW adaptation requires further examination.

\section{Acknowledgments}

This study was supported by the research fund (Project no. \#20088033-1) from the Ministry of Land, Transport and Maritime Affairs, Republic of Korea.

\section{References}

Butler AA and Le Roith D. 2001. Control of growth by the somatotrophic axis: growth hormone and the insulinlike growth factors have related and independent roles. Ann Rev Physiol 63, 141-164.

Froesch ER, Schmid CHR, Schwander J and Zapf J. 1985. Actions of insulin-like growth factors. Annu Rev Physiol 47, 443-467.

Inoue K and Takei Y. 2002. Diverse adaptability in Oryzias species to high environmental salinity. Zool Sci 19, 727-734.

Inoue $\mathrm{K}$ and Takei Y. 2003. Asian medaka fishes offer new models for studying mechanisms of seawater adaptation. Comp Biochem Physiol B 136, 635-645. 
Jones JI and Clemmons DR. 1995. Insulin-like growth factors and their binding proteins: biological actions. Endocr Rev 16, 3-34.

Kalujnaia S, McWilliam IS, Zaguinaiko VA, Feilen AL, Nicholson J, Hazon N, Cutler CP, Balment RJ, Cossins AR, Hughes M, Cramb G. 2007. Salinity adaptation and gene profiling analysis in the European eel (Anguilla anguilla) using microarray technology. Gen Comp Endocrinol 152, 274-280.

Kang CK, Tsai SC, Lee TH and Hwang PP. 2008. Differential expression of branchial $\mathrm{Na}+/ \mathrm{K}+$-ATPase of two medaka species, Oryzias latipes and Oryzias dancena, with different salinity tolerances acclimated to fresh water, brackish water and seawater. Comp Biochem Physiol A 151, 566-575.

Madsen SS and Bern HA. 1993. In vitro effects of insulinlike growth factor-I on gill $\mathrm{Na}^{+}, \mathrm{K}^{+}$-ATPase in coho salmon, Oncorhynchus kisutch. J Endocrinol 138, 23-30.

Mancera JM and McCormick SD. 1998. Evidence for growth hormone/insulin-like growth factor I axis regulation of seawater acclimation in the euryhaline teleost Fundulus heteroclitus. Gen Comp Endocrinol 111, 103-112.

McCormick SD. 1995. Hormonal control of gill $\mathrm{Na}^{+}, \mathrm{K}^{+}-$ ATPase and and chloride cell function. In: Wood CM and Shuttleworth TJ (Eds.), Fish Physiology Vol. XIV, Ionoregulation: Cellular and Molecular Approaches. Academic Press, New York, pp. 285-315.

McCormick SD. 1996. Effects of growth hormone and insulin-like growth factor I on salinity tolerance and gill $\mathrm{Na}^{+}, \mathrm{K}^{+}$-ATPase in Atlantic salmon (Salmo salar): interaction with cortisol. Gen Comp Endocrinol 101, 311.

McCormick SD. 2001. Endocrine control of osmoregulation in teleost fish. Am Zool 41, 781-794.

McCormick SD, Sakamoto T, Hasegawa S and Hirano T. 1991. Osmoregulatory actions of insulin-like growth factor-I in rainbow trout (Oncorhynchus mykiss). J Endocrinol 130, 87-92.

Meier KM, Figueiredo MA, Kamimura MT, Laurino J, Maggioni R, et al. 2009. Increased growth hormone $(\mathrm{GH})$, growth hormone receptor (GHR), and insulin- 'like growth factor I (IGF-I) gene transcription after hyperosmotic stress in the Brazilian flounder Paralichthys orbignyanus. Fish Physiol Biochem 35, 501-509.

Ponce M, Infante C, Funes V and Manchado M. 2008. Molecular characterization and gene expression analysis of insulin-like growth factors I and II in the redbanded seabream, Pagrus auriga: transcriptional regulation by growth hormone. Comp Biochem Physiol B 150, 418-426.

Reinecke, M., 2006. Insulin-like growth factor I and II in fish. In: Reinecke M, Zaccone G and Kapoor BG (Eds.), Fish Endocrinology, vol. I. Science Publishers, New Hampshire, pp. 87-130.

Reinecke M, Björnsson BT, Dickhoff WW, McCormick SD, Navarro I, Power DM, Gutiérrez J. 2005. Growth hormone and insulin-like growth factors: Where we are and where to go. Gen Comp Endocrinol 135, 20-24.

Roberts TR. 1998. Systematic observations on tropical Asian medakas or ricefishes of the genus Oryzias with descriptions of four new species. Ichthyol. Res 45, 213224.

Sakamoto T and Hirano T. 1993. Expression of insulin-like growth factor I gene in osmoregulatory organs during seawater adaptation of the salmonid fish: possible mode of osmoregulatory action of growth hormone. Proc Natl Acad Sci USA 90, 1912-1916.

Shamblott MJ, Cheng CM, Bolt D and Chen TT. 1995. Appearance of insulin-like growth factor mRNA in the liver and pyloric ceca of a teleost in response to exogenous growth hormone. Proc Natl Acad Sci USA 92, 6943-6946.

Wittbrodt J, Shima A and Schartl M. 2002. Medaka - a model organism from the far East. Nat Rev Genet 3, 5364.

Yada T. 2007. Growth hormone and fish immune system. Gen Comp Endocrinol 152, 353-358.

(Received 14 July 2010; Revised 3 September 2010; Accepted 11 September 2010) 